\title{
molecules
}

ISSN 1420-3049

www.mdpi.com/journal/molecules

Article

\section{Pinelliae Rhizoma, a Toxic Chinese Herb, Can Significantly Inhibit CYP3A Activity in Rats}

\author{
Jinjun Wu ${ }^{1, \dagger}$, Zaixing Cheng ${ }^{2, \dagger}$, Shugui He ${ }^{1}$, Jian Shi ${ }^{1}$, Shuqiang Liu ${ }^{1}$, Guiyu Zhang ${ }^{1}$, \\ Lijun Zhu ${ }^{1}$, Liang Liu ${ }^{3}$, Zhongqiu Liu ${ }^{1}$, Na Lin ${ }^{1,4}$ and Linlin Lu ${ }^{1, *}$
}

1 International Institute for Translational Chinese Medicine, Guangzhou University of Chinese Medicine, Guangzhou 510006, China; E-Mails: wujinjun1018@163.com (J.W.); heshugui666@163.com (S.H.); shijian0225@126.com (J.S.); lshq120@163.com (S.L.); zhangguiyu0226@126.com (G.Z.); zhulijun115623@163.com (L.Z.); liuzq@gzucm.edu.cn (Z.L.); linna888@163.com (N.L.)

2 College of Pharmacy, Fujian University of Traditional Chinese Medicine, Fuzhou 350108, China; E-Mail: chengzaixing2086@sohu.com

3 State Key Laboratory of Quality Research in Chinese Medicine, Macau University of Science and Technology, Macau, China; E-Mail: 1liu@must.edu.mo

4 Institute of Chinese Meteria Medica, China Academy of Chinese Medical Sciences, Beijing 100700, China

$\dagger$ These authors contributed equally to this work.

* Author to whom correspondence should be addressed; E-Mail: 1llu@gzucm.edu.cn; Tel.: +86-20-3935-7902; Fax: +86-20-3935-8071.

Academic Editor: Marcello Iriti

Received: 24 November 2014 / Accepted: 31 December 2014 / Published: 7 January 2015

\begin{abstract}
Raw Pinelliae Rhizoma (RPR) is a representative toxic herb that is widely used for eliminating phlegm or treating cough and vomiting. Given its irritant toxicity, its processed products, including Pinelliae Rhizoma Praeparatum (PRP) and Pinelliae Rhizoma Praeparatum cum Zingibere et Alumine (PRPZA), are more commonly applied and administered concomitantly with other chemical drugs, such as cough medications. This study aimed to investigate the effects of RPR, PRP, and PRPZA on CYP3A activity. Testosterone (Tes) and buspirone (BP) were used as specific probe substrates ex vivo and in vivo, respectively. CYP3A activity was determined by the metabolite formation ratios from the substrates. Ex vivo results show that the metabolite
\end{abstract}


formation ratios from Tes significantly decreased, indicating that RPR, PRP, and PRPZA could inhibit CYP3A activity in rats. CYP3A protein and mRNA levels were determined to explore the underlying mechanism. These levels showed marked and consistent down-regulation with CYP3A activity. A significant decrease in metabolite formation ratios from BP was also found in PRPZA group in vivo, implying that PRPZA could inhibit CYP3A activity. Conclusively, co-administration of PR with other CYP3A-metabolizing drugs may cause drug-drug interactions. Clinical use of PR-related formulae should be monitored carefully to avoid adverse interactions.

Keywords: Pinelliae Rhizoma; CYP3A; testosterone; buspirone; drug-drug interactions

\section{Introduction}

Pinelliae Rhizoma (PR), the tuber of Pinellia ternate (Thunb.) Breit, is a commonly used Chinese herb with high bioactivity against cough and vomiting, or for eliminating the stagnation of phlegm [1-3]. PR is distributed in China, Korea, and Japan. It has been used as an essential drug in Chinese clinics for thousands of years. However, PR is also a representative toxic herbal medicine, which can cause irritant toxicity to oral, throat, and gastrointestinal mucosa [4-6]. Thus, PR is commonly used in its preparation forms in Chinese clinics. As recorded in the Chinese Pharmacopoeia (2005 and 2010 Edition), PR mainly comprises raw Pinelliae Rhizoma (RPR), Pinelliae Rhizoma Praeparatum (PRP), and Pinelliae Rhizoma Praeparatum cum Zingibere et Alumine (PRPZA) according to the different processing methods for toxicity reduction and pharmacological effect improvement. PRP is the product of RPR processed with alkaline solution and licorice. PRPZA is the product of RPR processed with alum and ginger juice. These excipients applied during processing can effectively eliminate or reduce irritant toxicity by decreasing the amount of needle-like raphides in RPR, which are popularly considered to be a major irritant component [7-9].

Although a large number of trials of PR have been carried out, the exact mechanism of its toxicity, especially its irritant effects, remains unclear. PR consists of multiple components, most of which are not easy to analyze. Modern phytochemical studies showed that the content of starch in PR is as high as $75 \%$ [10]. In addition, alkaloids, essential oils, amino acids, organic acids, and proteins are the chemical components of PR [7,11-13]. Unfortunately, the specific purified compounds derived from the herb were still not obtained. Previously, we had determined the abundant alkaloid trigonelline in coffee beans [14]. Trigonelline, distributed widely in plants, is also a major alkaloid in PR, but is not the toxic component. Other studies reported about PR's toxic components and obtained different results, which have still not been recognized by authorities $[10,15]$. The raphides in RPR are currently regarded as a major irritant component; they are composed of calcium oxalate, protein, and trace amounts of polysaccharides [16]. Given the wide application of PR in Chinese clinical practice and unavailable studies about its exact toxicity mechanism, further research must be conducted to explore the underlying mechanism and provide useful information for a better understanding of PR.

Combination therapy is advocated based on the clinical settings and properties of each herb. For toxic herbs, co-therapy can help counteract their toxicities or side effects and simultaneously enhance their 
therapeutic effects $[17,18]$. PR, a representative toxic herbal medicine, can cause irritant toxicity when used as its raw form. Thus, RPR is commonly applied in its preparation forms, such as PRP or PRPZA, and frequently co-administered concomitantly with other chemical drugs because patients with cough, vomiting, or stagnation of phlegm require multiple drug therapy. Currently, PR and many chemical drugs are co-used to treat these diseases; these drugs include oxycodone [19], codeine [20], dextromethorphan [21], domperidone [22], chlorpromazine [23], and ambroxol [24], etc., which are primarily or partially metabolized by cytochrome P450 isoenzyme 3A (CYP3A). Unfortunately, no study has reported the influence of PR on CYP3A. The safety assessment related to drug-drug interactions (DDIs) between PR and CYP3A-metabolizing drugs is essential and urgent.

In recent years, numerous DDIs between herbal medicines and chemical drugs have been documented [25-27]. The activity and expression of cytochrome P450 enzymes are closely related to DDIs. DDIs usually occur because of the inhibition or induction of CYPs. For example, the area under the plasma concentration-time curve and blood concentrations of cyclosporine, midazolam, tacrolimus, amitriptyline, digoxin, indinavir, warfarin, phenprocoumon, and theophylline can be significantly reduced after co-administration of St. John's wort (Hypericum perforatum) via CYP3A induction [28]. CYP inhibition results in an undesirable elevation in plasma concentrations of co-administered drugs, causing adverse effects and toxicologically unsafe consequences [29]. CYP3A is the most abundant P450 enzyme in the liver, which is involved in the metabolism of $40 \%-50 \%$ of all currently used drugs [30]. However, CYP3A activity is frequently affected by its own substrates, and is recognized as one of the key factors causing DDIs [31]. In clinics, PR is frequently co-used with other herbal medicine or chemical drugs for treating diseases. Thus, the potential risk of DDIs between PR and other co-used drugs must be evaluated to provide important guidance for the clinical practice of PR.

In the present study, CYP3A activity ex vivo and in vivo was examined after rats were pretreated with RPR, PRP, or PRPZA for $7 \mathrm{~d}$ using testosterone (Tes) and buspirone (BP), respectively, which have been accepted as specific probe substrates for CYP3A activity by the U.S. Food and Drug Administration (USFDA). Tes is primarily metabolized by CYP3A into 6 $\beta$-hydroxytestosterone (6 $\beta$-OH-Tes) (Figure 1) [32]. CYP3A activity in rat liver microsomes (RLMs) was determined by measuring the formation rate of $6 \beta-\mathrm{OH}-\mathrm{Tes}$ using a validated ultra-performance liquid chromatography (UPLC) method [33,34]. BP is mainly metabolized by CYP3A into 1-(2-pyrimidinyl) piperazine (1-PP) and 6'-hydroxybuspirone (6'-OH-BP) (Figure 2) [35]. The pharmacokinetic characteristics of BP after pretreatment with PR were investigated. The concentrations of BP, 1-PP, and 6'-OH-BP in rat plasma were determined via a validated UPLC-tandem mass spectrometry method. Furthermore, western blot and real-time PCR were applied to explore the underlying mechanism of the changes in CYP3A activity.

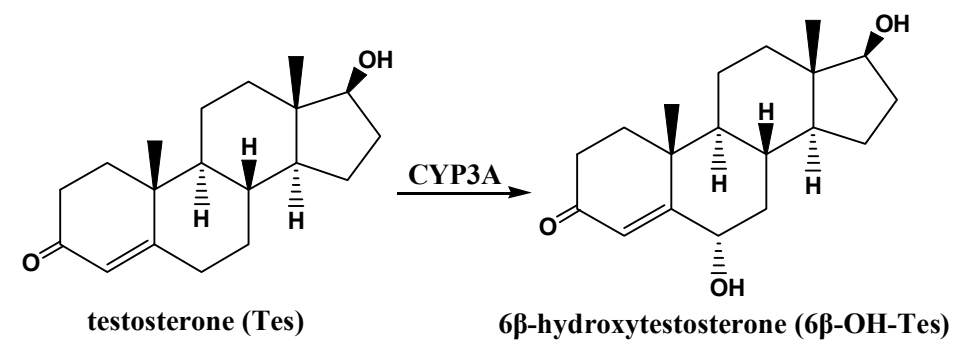

Figure 1. The major metabolic pathway of testosterone [32]. 


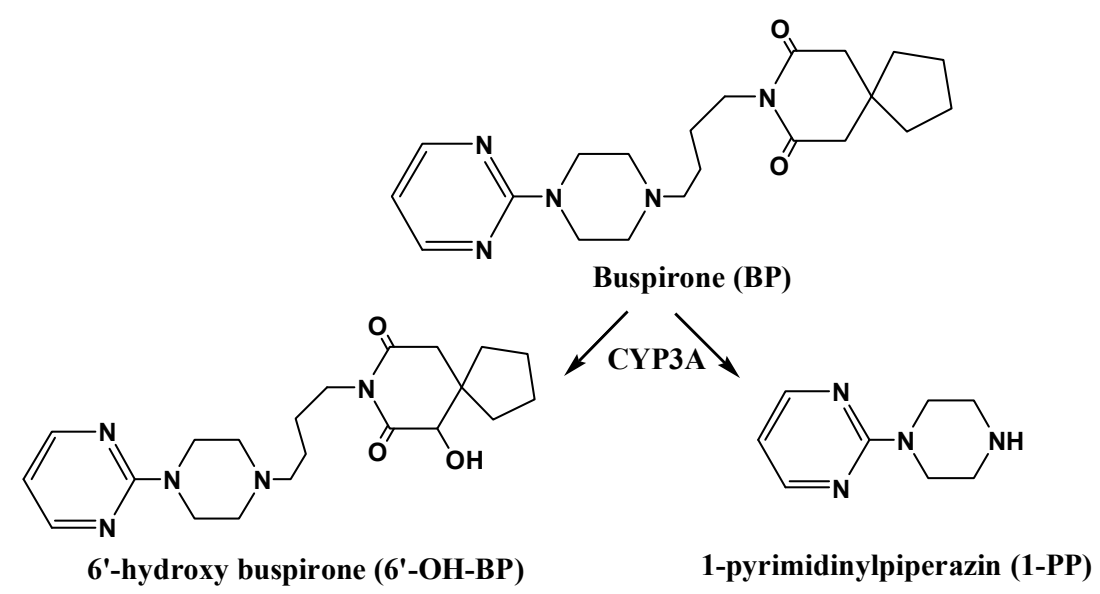

Figure 2. The major metabolic pathways of buspirone [35].

\section{Results and Discussion}

\subsection{Effects of RPR, PRP or PRPZA on Hepatic CYP3A Activity ex Vivo}

\subsubsection{Effects of RPR on Hepatic CYP3A Activity ex Vivo}

Figure 3A shows the hepatic CYP3A activity observed in RLMs after 7 days of pretreatment with RPR $\left(0.45,0.9\right.$ or $1.8 \mathrm{~g} \cdot \mathrm{kg}^{-1} \cdot \mathrm{day}^{-1}$, p.o.) or saline. Compared with that in the control group, the formation rates of $6 \beta-\mathrm{OH}-\mathrm{Tes}$ from 4,10 , and $30 \mu \mathrm{mol} \cdot \mathrm{L}^{-1}$ Tes in the $0.45 \mathrm{~g} \cdot \mathrm{kg}^{-1} \cdot \mathrm{day}^{-1}$ dose group decreased by $14 \%(p<0.05), 12 \%(p<0.05)$, and $4 \%(p>0.05)$, respectively; those in the $0.9 \mathrm{~g} \cdot \mathrm{kg}^{-1} \cdot \mathrm{day}^{-1}$ dose group decreased by $24 \%(p<0.05), 19 \%(p<0.05)$, and $11 \%(p<0.05)$, respectively; those in the $1.8 \mathrm{~g} \cdot \mathrm{kg}^{-1} \cdot$ day $^{-1}$ group decreased by $45 \%(p<0.05), 36 \%(p<0.05)$, and $32 \%(p<0.05)$, respectively.

\subsubsection{Effects of PRP on Hepatic CYP3A Activity ex Vivo}

Figure 3B shows the hepatic CYP3A activity observed in RLMs after 7 days of pretreatment with PRP $\left(0.45,0.9\right.$ or $1.8 \mathrm{~g} \cdot \mathrm{kg}^{-1} \cdot \mathrm{day}^{-1}$, p.o.) or saline. Compared with that in the control group, the formation rates of $6 \beta-\mathrm{OH}-\mathrm{Tes}$ from 4,10 , and $30 \mu \mathrm{mol} \cdot \mathrm{L}^{-1}$ Tes in the $0.45 \mathrm{~g} \cdot \mathrm{kg}^{-1} \cdot \mathrm{day}^{-1}$ dose group decreased by $29 \%(p<0.05), 29 \%(p<0.05)$, and $31 \%(p<0.05)$, respectively; those in the $0.9 \mathrm{~g} \cdot \mathrm{kg}^{-1} \cdot \mathrm{day}^{-1}$ dose group decreased by $32 \%(p<0.05), 27 \%(p<0.05)$, and $23 \%(p<0.05)$, respectively; those in the $1.8 \mathrm{~g} \cdot \mathrm{kg}^{-1} \cdot \mathrm{day}^{-1}$ dose group decreased by $24 \%(p<0.05), 28 \%(p<0.05)$, and $18 \%(p<0.05)$, respectively.

\subsubsection{Effects of PRPZA on Hepatic CYP3A Activity ex Vivo}

Figure 3C shows the hepatic CYP3A activity observed in RLMs after 7 days of pretreatment with PRPZA $\left(0.45,0.9\right.$ or $1.8 \mathrm{~g} \cdot \mathrm{kg}^{-1} \cdot \mathrm{day}^{-1}$, p.o.) or saline. Compared with that in the control group, the formation rates of $6 \beta-\mathrm{OH}-\mathrm{Tes}$ from 4,10 , and $40 \mu \mathrm{mol} \cdot \mathrm{L}^{-1}$ Tes in the $0.45 \mathrm{~g} \cdot \mathrm{kg}^{-1} \cdot \mathrm{day}^{-1}$ dose group decreased by $16 \%(p<0.05), 1 \%(p>0.05)$, and $1 \%(p>0.05)$, respectively; those in the $0.9 \mathrm{~g} \cdot \mathrm{kg}^{-1} \cdot \mathrm{day}^{-1}$ dose group decreased by $21 \%(p<0.05), 16 \%(p<0.05)$, and $9 \%(p<0.05)$, respectively; those in the $1.8 \mathrm{~g} \cdot \mathrm{kg}^{-1} \cdot$ day $^{-1}$ dose group decreased by $23 \%(p<0.05), 9 \%(p>0.05)$, and $10 \%(p<0.05)$, respectively. 

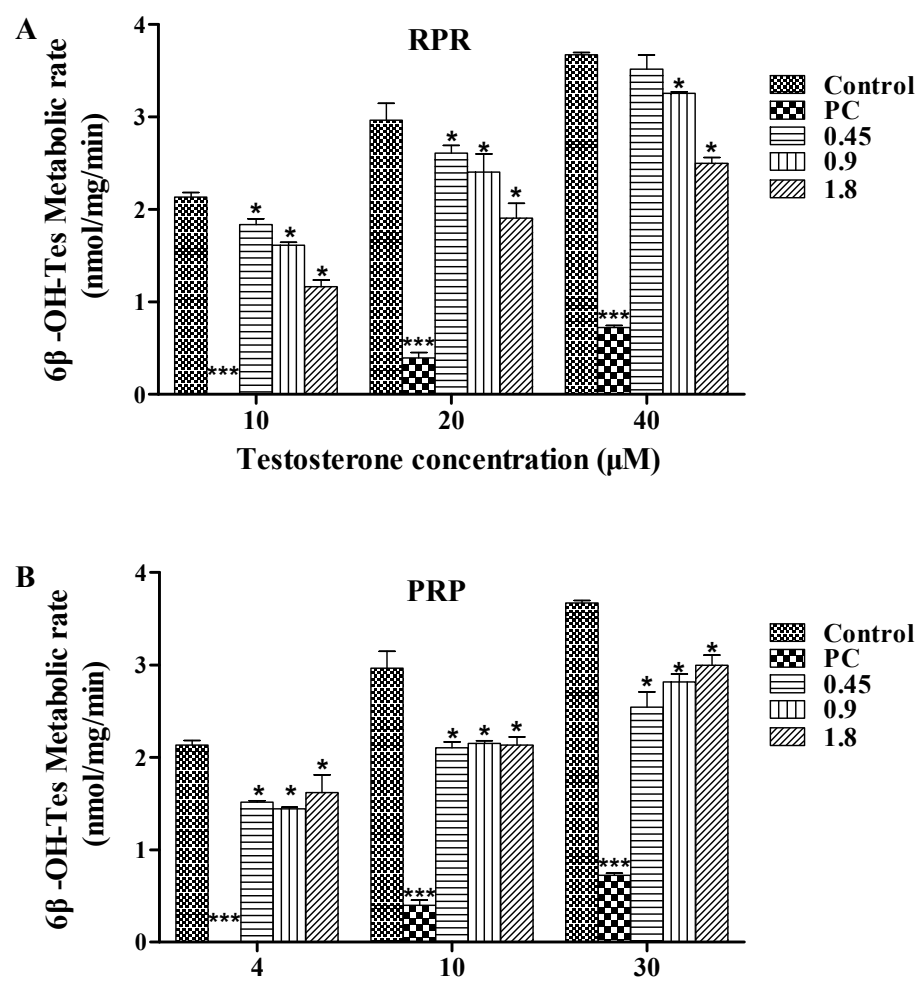

Testosterone concentration $(\mu \mathrm{M})$

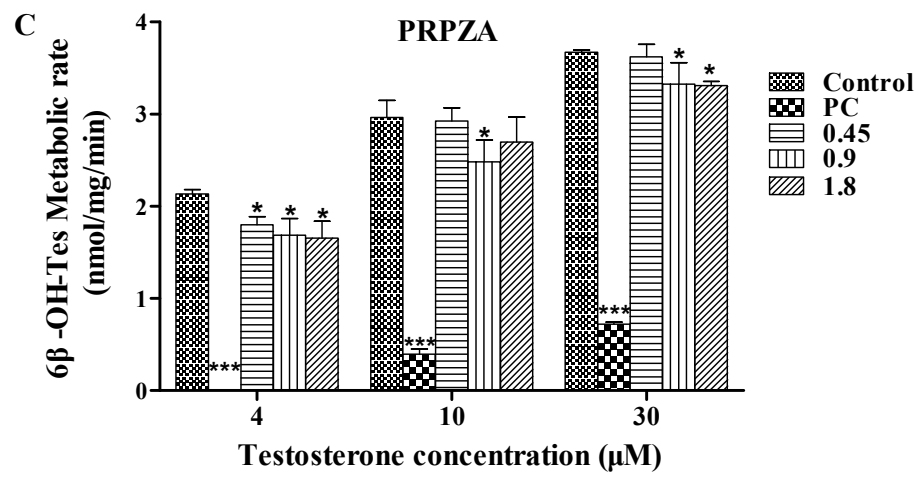

Figure 3. Formation rates of $6 \beta$-hydroxytestosterone ( $6 \beta-\mathrm{OH}-\mathrm{Tes})$ from testosterone (Tes) in rat liver microsomes (RLMs). Before the RLMs were prepared, rats were orally pretreated with RPR (A); PRP (B); PRPZA (C) $\left(\mathrm{g} \cdot \mathrm{kg}^{-1} \cdot \mathrm{day}^{-1}\right)$, or saline once daily for 7 days. The rats were orally pretreated with SKF-525A $\mathrm{HCl}\left(0.1 \mathrm{~g} \cdot \mathrm{kg}^{-1}, 7\right.$ days $)$ as the positive control (PC) for CYP3A inhibition. * $p<0.05$ and $* * * p<0.001$ compared with the control group. Data are expressed as mean $\pm \mathrm{SD}(n=3)$.

As shown in Figure 3, the formation ratio of $6 \beta-\mathrm{OH}-\mathrm{Tes}$ from Tes significantly decreased in the treated groups compared with that in the control group, suggesting that RPR, PRP, and PRPZA could inhibit CYP3A activity. Based on the results, we also found that different but regular inhibitory effects caused by PR were related to its processed forms. RPR at $1.8 \mathrm{~g} \cdot \mathrm{kg}^{-1} \cdot \mathrm{day}^{-1}$ had the strongest inhibitory effects on CYP3A activity ex vivo. The processed products, especially PRPZA, only slightly inhibited CYP3A activity. Furthermore, some treated groups showed dosage dependency of the inhibitory effects. The inhibitory effects on CYP3A activity increased gradually with increasing dose of RPR. The PRP-treated groups showed an opposite tendency at $30 \mu \mathrm{mol} \cdot \mathrm{L}^{-1}$ Tes that the inhibitory effects 
decreased gradually with rising dose. The PRPZA groups did not show marked dose-dependent inhibition on CYP3A activity. Therefore, the different processing methods of PR and different administration doses produced various but regular inhibitory effects on CYP3A. These findings may provide useful guidance for the clinical application of PR.

\subsection{Effects of RPR, PRP or PRPZA on Hepatic CYP3A Protein and mRNA Expression Levels}

\subsubsection{Effects of RPR, PRP or PRPZA on Hepatic CYP3A Protein Expression Levels}

Figure 4A shows hepatic CYP3A protein expression levels in RLMs after 7 days of oral pretreatment with RPR/PRP/PRPZA $\left(0.45,0.9\right.$ or $\left.1.8 \mathrm{~g} \cdot \mathrm{kg}^{-1} \cdot \mathrm{day}^{-1}\right)$, or saline. The ratio of CYP3A to $\beta$-actin concentration was used as the relative protein expression level. Compared with the control group, the CYP3A protein expression levels in the RPR group at $0.45,0.9$ and $1.8 \mathrm{~g} \cdot \mathrm{kg}^{-1} \cdot \mathrm{day}^{-1} \mathrm{doses}$ decreased by $42 \%(p<0.01), 52 \%(p<0.01)$ and $60 \%(p<0.001)$, respectively; those in the PRP group decreased by $43 \%(p<0.01), 41 \%(p<0.01)$ and $31 \%(p<0.01)$, respectively; those in the PRPZA group decreased by $34 \%(p<0.01), 31 \%(p<0.01)$ and $44 \%(p<0.01)$, respectively.

\subsubsection{Effects of RPR, PRP or PRPZA on Hepatic CYP3A1 mRNA Expression Levels}

Figure 4B shows hepatic CYP3A1 mRNA expression levels in rat livers after 7 days of the same pretreatment. CYP3A1 mRNA expression levels were determined by measuring the ratio of CYP3A1 to GAPDH. Compared with the control group, the CYP3A1 mRNA expression levels in the RPR group at $0.45,0.9$ and $1.8 \mathrm{~g} \cdot \mathrm{kg}^{-1} \cdot \mathrm{day}^{-1}$ doses decreased by $61 \%(p<0.001), 69 \%(p<0.001)$ and $53 \%$ $(p<0.001)$, respectively; those in the PRP group decreased by $22 \%(p<0.01), 27 \%(p<0.001)$ and $42 \%(p<0.001)$, respectively; those in the PRPZA group decreased by $47 \%(p<0.001), 57 \%$ $(p<0.001)$ and $66 \%(p<0.001)$, respectively.

\subsubsection{Effects of RPR, PRP or PRPZA on Hepatic CYP3A2 mRNA Expression Levels}

Figure 4C shows hepatic CYP3A2 mRNA expression levels in rat livers after 7 days of the same pretreatment. CYP3A2 mRNA expression levels were determined by measuring the ratio of CYP3A2 to GAPDH. Compared with the control group, the CYP3A2 mRNA expression levels in the RPR group at $0.45,0.9$ and $1.8 \mathrm{~g} \cdot \mathrm{kg}^{-1} \cdot \mathrm{day}^{-1}$ doses decreased by $37 \%(p<0.001), 32 \%(p<0.001)$ and $42 \%$ $(p<0.001)$, respectively; those in the PRP group decreased by $-0.2 \%(p>0.05), 6 \%(p>0.05)$ and $23 \%(p<0.01)$, respectively; those in the PRPZA group decreased by 36\% $(p<0.001), 37 \%$ $(p<0.001)$ and $37 \%(p<0.001)$, respectively.

CYP3A protein and mRNA levels were determined to explore the underlying mechanism of changes in CYP3A activity. The CYP3A isoforms in rat include CYP3A1, CYP3A2, and CYP3A23. CYP3A1 and CYP3A2 are the most versatile isoforms in rat liver, which is involved in the metabolism of currently used drugs. Thus, in this research, we focused on CYP3A1/2 in rats. However, given the high homology of CYP3A1 and CYP3A2 [36], we investigated the CYP3A protein and CYP3A1/2 mRNA expression levels. The results show that RPR, PRP, and PRPZA significantly down-regulated the CYP3A protein and CYP3A1/2 mRNA expression levels. We confirmed that CYP3A activity decreased because of the down-regulated expression levels of CYP3A protein and mRNA. 

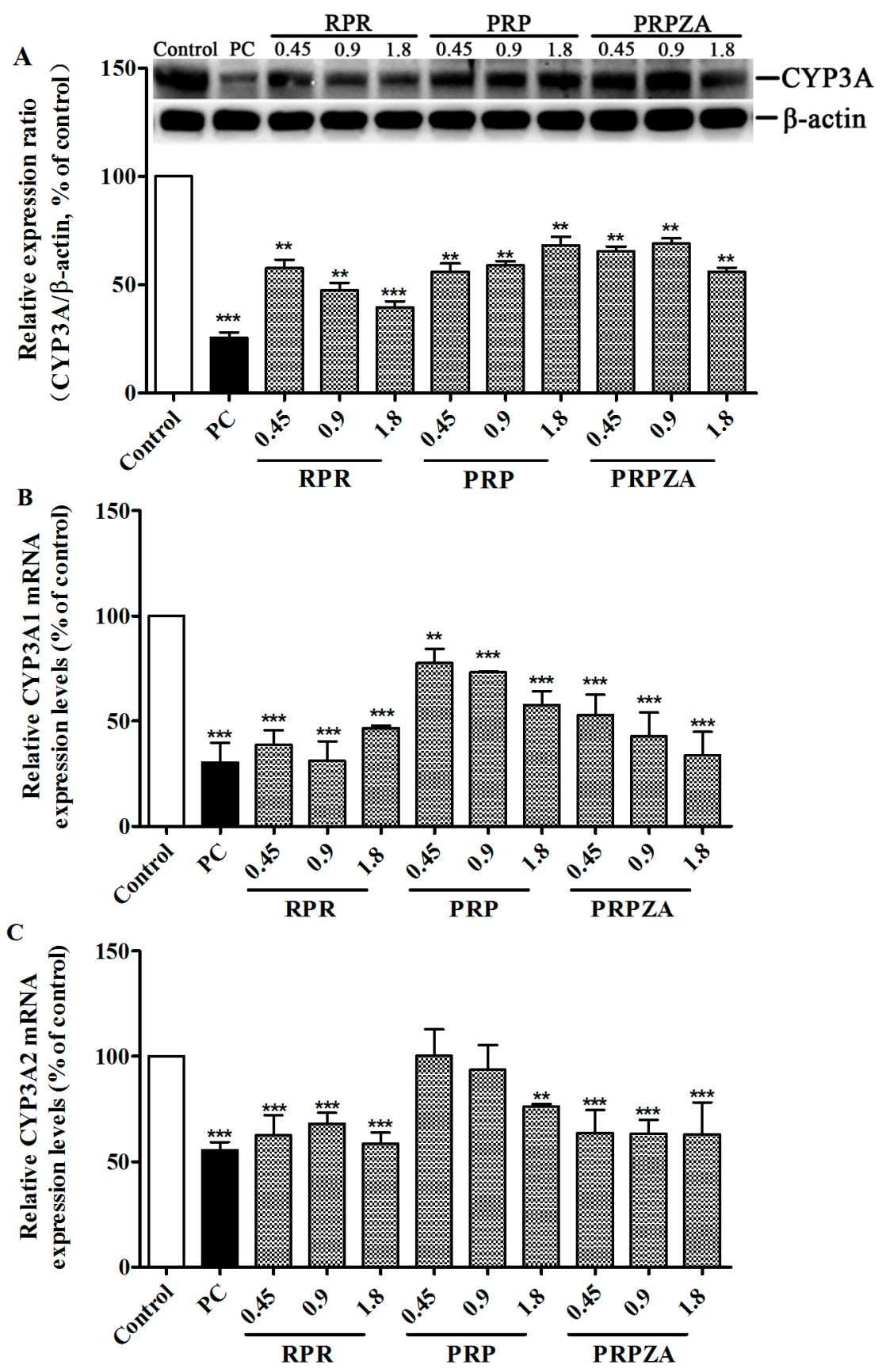

Figure 4. CYP3A protein (A); CYP3A1 mRNA (B) and CYP3A2 mRNA (C) expression levels in rat livers. Before the rat livers were prepared, rats were orally pretreated with RPR (A); PRP (B); PRPZA (C) $\left(\mathrm{g} \cdot \mathrm{kg}^{-1} \cdot \mathrm{day}^{-1}\right)$, or saline once daily for 7 days. The rats were orally pretreated with SKF-525A HCl $\left(0.1 \mathrm{~g} \cdot \mathrm{kg}^{-1}, 7\right.$ days $)$ as the positive control (PC) for CYP3A inhibition. $* * p<0.01$ and $* * * p<0.001$ compared with the control group. Data are expressed as mean $\pm \mathrm{SD}(n=3)$.

\subsection{Effects of RPR, PRP or PRPZA on BP Pharmacokinetics in Vivo}

Based on the results ex vivo, we chose the medium dose $\left(0.9 \mathrm{~g} \cdot \mathrm{kg}^{-1} \cdot \mathrm{day}^{-1}\right)$ as the treated dose to further study the influence of PR on CYP3A activity via BP metabolism in vivo. Figure 5 shows the mean plasma concentration-time profiles of BP, 1-PP, and 6'-OH-BP after oral pretreatment with RPR, PRP, PRPZA, or saline for 7 days before intravenous injection of $0.5 \mathrm{~g} \cdot \mathrm{kg}^{-1} \mathrm{BP} \cdot \mathrm{HCl}$. As shown in Table 1, 
the formation ratio of 6'-OH-BP from BP ( $\mathrm{AUC}_{0-\mathrm{t}}$ of 6'-OH-BP / $\mathrm{AUC}_{0-\mathrm{t}}$ of $\mathrm{BP}$ ) in the control group was $0.24 \pm 0.06$ [37], whereas those in the RPR, PRP and PRPZA groups were $0.24 \pm 0.08,0.28 \pm 0.11$ and $0.25 \pm 0.05$, respectively. The formation ratio of 1-PP from BP ( $\mathrm{AUC}_{0-\mathrm{t}}$ of 1-PP/AUC0-t of BP) in the control group was $0.30 \pm 0.11$ [37], whereas those in the RPR, PRP and PRPZA groups were $0.22 \pm 0.04,0.31 \pm 0.08$ and $0.15 \pm 0.03$, respectively. Compared with that in the control group, the formation ratio of 1-PP from BP significantly decreased by 50\% $(p<0.01)$ in the PRPZA group; other formation ratios of 6'-OH-BP or 1-PP from BP showed no evident change $(p>0.05)$.
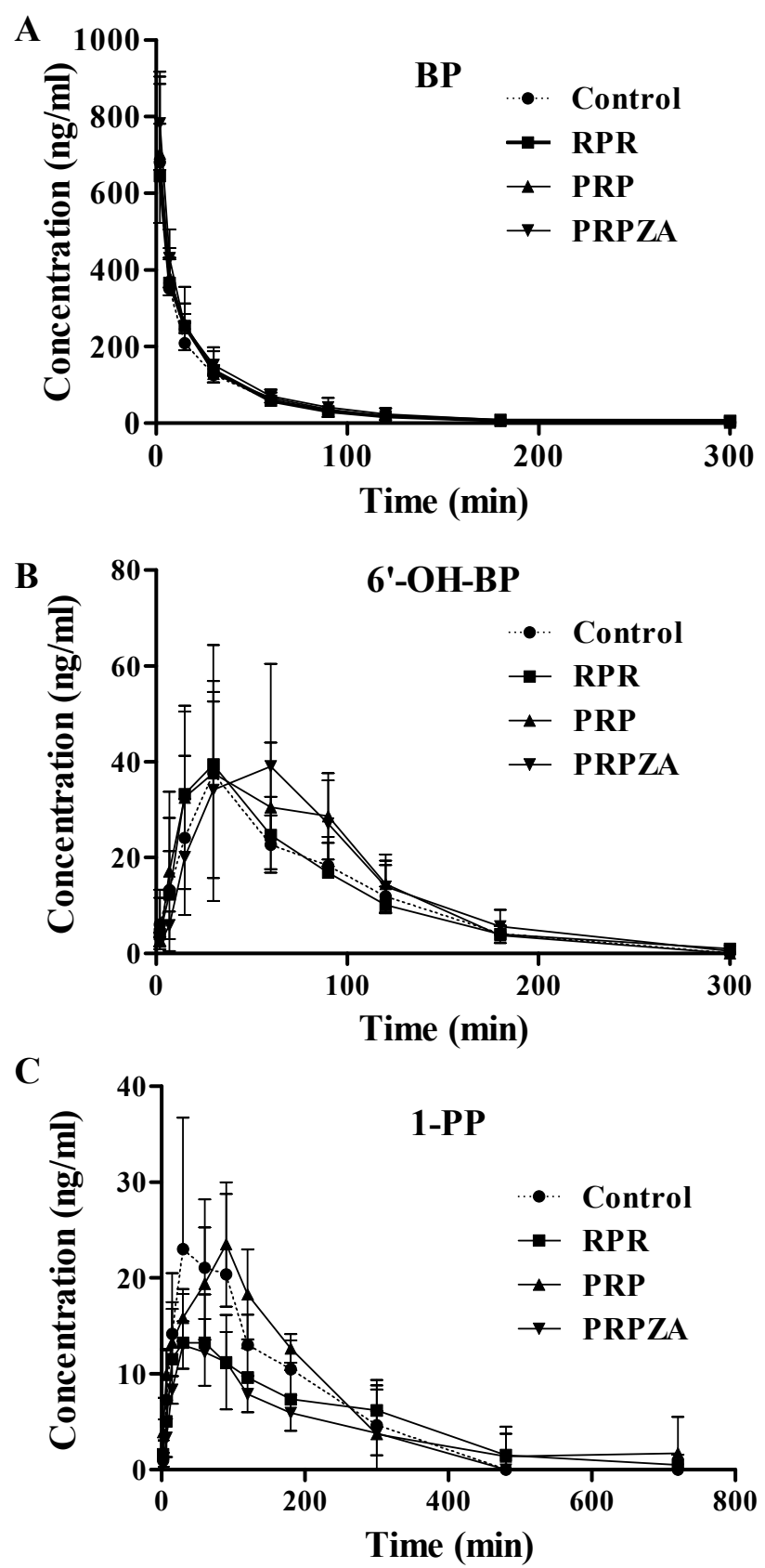

Figure 5. Concentration-time profiles of buspirone (BP) (A), 6'-hydroxybuspirone (6'-OH-BP) (B), and 1-(2-pyrimidinyl) piperazine (1-PP) (C) in rats after intravenous injection of $0.5 \mathrm{mg} \cdot \mathrm{kg}^{-1} \mathrm{BP} \cdot \mathrm{HCl}$. Prior to intravenous injection of $\mathrm{BP} \cdot \mathrm{HCl}$, the rats were orally pretreated with RPR, PRP, PRPZA, or saline once daily for 7 days. The data presented in this figure indicate mean $\pm \mathrm{SD}(n=5)$. 
Table 1. Pharmacokinetic parameters of buspirone (BP), 1-(2-pyrimidinyl) piperazine (1-PP) and 6'-hydroxybuspirone (6'-OH-BP) in rats after intravenous injection of $0.5 \mathrm{mg} \cdot \mathrm{kg}^{-1} \mathrm{BP} \cdot \mathrm{HCl}$. Prior to intravenous injection of $\mathrm{BP} \cdot \mathrm{HCl}$, the rats were orally pretreated with RPR, PRP, PRPZA $\left(0.9 \mathrm{~g} \cdot \mathrm{kg}^{-1} \cdot \mathrm{day}^{-1}\right)$, or saline once daily for 7 days. The data presented in this table indicate mean $\pm \mathrm{SD}(n=5)$.

\begin{tabular}{|c|c|c|c|c|}
\hline Variable & Control & RPR & PRP & PRPZA \\
\hline \multicolumn{5}{|l|}{ BP } \\
\hline$A U C_{0-\mathrm{t}}\left(\mu \mathrm{g} \cdot \mathrm{min} \cdot \mathrm{mL}^{-1}\right)$ & $14,774.65 \pm 2767.74$ & $16,253.05 \pm 4045.20$ & $16,146.91 \pm 953.18$ & $17,502.24 \pm 4206.67$ \\
\hline$C_{\max }\left(\mathrm{ng} \cdot \mathrm{mL}^{-1}\right)$ & $681.16 \pm 253.80$ & $672.76 \pm 113.10$ & $702.01 \pm 181.79$ & $783.03 \pm 121.97$ \\
\hline$t_{1 / 2}(\min )$ & $118.15 \pm 9.83$ & $150.54 \pm 34.79$ & $146.12 \pm 15.33$ & $96.79 \pm 8.02$ \\
\hline$C L\left(\mathrm{~mL} \cdot \mathrm{min}^{-1} \cdot \mathrm{kg}^{-1}\right)$ & $34.37 \pm 6.05$ & $32.01 \pm 7.99$ & $30.53 \pm 1.90$ & $29.74 \pm 7.03$ \\
\hline$V_{\mathrm{d}}\left(\mathrm{L} \cdot \mathrm{kg}^{-1}\right)$ & $5806.61 \pm 752.26$ & $7263.06 \pm 3444.26$ & $6436.20 \pm 729.96$ & $4175.27 \pm 1125.25$ \\
\hline \multicolumn{5}{|l|}{ 6'-OH-BP } \\
\hline$A U C_{0-\mathrm{t}}\left(\mu \mathrm{g} \cdot \min \cdot \mathrm{mL}^{-1}\right)$ & $3515.02 \pm 1032.97$ & $3741.64 \pm 811.23$ & $4405.57 \pm 1757.76$ & $4468.04 \pm 1787.44$ \\
\hline$C_{\max }\left(\mathrm{ng} \cdot \mathrm{mL}^{-1}\right)$ & $38.96 \pm 17.86$ & $40.05 \pm 16.09$ & $43.66 \pm 22.07$ & $41.43 \pm 18.16$ \\
\hline$t_{1 / 2}(\min )$ & $43.62 \pm 11.61$ & $62.62 \pm 21.72$ & $38.23 \pm 9.75$ & $40.78 \pm 10.74$ \\
\hline$t_{\max }(\min )$ & $42.00 \pm 26.83$ & $27.00 \pm 6.71$ & $51.00 \pm 36.12$ & $60.00 \pm 21.21$ \\
\hline$C L\left(\mathrm{~mL} \cdot \mathrm{min}^{-1} \cdot \mathrm{kg}^{-1}\right)$ & $151.43 \pm 39.84$ & $139.88 \pm 36.57$ & $131.59 \pm 58.71$ & $126.79 \pm 49.62$ \\
\hline$V_{\mathrm{d}}\left(\mathrm{L} \cdot \mathrm{kg}^{-1}\right)$ & $9667.04 \pm 4169.74$ & $13,323.56 \pm 7985.70$ & $7388.06 \pm 3679.70$ & $7013.16 \pm 1855.36$ \\
\hline 6'-OH-BP/BP ratio & $0.24 \pm 0.06$ & $0.24 \pm 0.08$ & $0.28 \pm 0.11$ & $0.25 \pm 0.05$ \\
\hline \multicolumn{5}{|l|}{ 1-PP } \\
\hline$A U C_{0-\mathrm{t}}\left(\mu \mathrm{g} \cdot \min \cdot \mathrm{mL}^{-1}\right)$ & $4249.31 \pm 1290.50$ & $3624.86 \pm 1142.96$ & $4958.20 \pm 1259.90$ & $2596.72 \pm 557.34$ \\
\hline $\mathrm{C}_{\max }\left(\mathrm{ng} \cdot \mathrm{mL}^{-1}\right)$ & $27.35 \pm 12.57$ & $17.35 \pm 7.20$ & $25.99 \pm 4.86$ & $13.93 \pm 3.87$ \\
\hline$t_{1 / 2}(\min )$ & $160.16 \pm 52.85$ & $244.73 \pm 76.46$ & $280.52 \pm 308.26$ & $268.82 \pm 55.17$ \\
\hline$t_{\max }(\min )$ & $48.00 \pm 26.83$ & $51.00 \pm 29.24$ & $78.00 \pm 16.43$ & $54.00 \pm 25.10$ \\
\hline$C L\left(\mathrm{~mL} \cdot \mathrm{min}^{-1} \cdot \mathrm{kg}^{-1}\right)$ & $126.20 \pm 36.57$ & $143.18 \pm 56.52$ & $97.07 \pm 39.69$ & $198.43 \pm 34.45$ \\
\hline$V_{\mathrm{d}}\left(\mathrm{L} \cdot \mathrm{kg}^{-1}\right)$ & $30,074.20 \pm 16,188.54$ & $46,942.34 \pm 11,037.16$ & $25,588.78 \pm 11,512.25$ & $69,192.39 \pm 18,332.26$ \\
\hline 1-PP/BP ratio & $0.30 \pm 0.11$ & $0.22 \pm 0.04$ & $0.31 \pm 0.08$ & $0.15 \pm 0.03 * *$ \\
\hline
\end{tabular}

The BP pharmacokinetic behaviors showed that the formation ratio of 1-PP from BP significantly decreased in the PRPZA group, but other formation ratios of 6'-OH-BP or 1-PP from BP did not markedly change. This finding demonstrates that PRPZA could inhibit CYP3A activity, but RPR and PRP had no significant effects on CYP3A activity in vivo. Conclusively, the inhibitory effects of RPR and PRP ex vivo were more evident than those in vivo. We presume that the inconsistent effects may be mainly attributed to the complex environment of rat in vivo, which will be explored urgently in the next research.

\section{Experimental Section}

\subsection{Chemicals and Reagents}

Buspirone $\mathrm{HCl}$, testosterone (purity $>98 \%$ ), 6 6 -hydroxytestosterone (purity $>98 \%$ ), and Gemfibrozil (used as an internal standard, purity $>98 \%$ ) were purchased from Sigma-Aldrich 
(St. Louis, MO, USA). 1-(2-pyrimidinyl) piperazine, 6'-hydroxybuspirone and SKF-525A HCl (used as a positive control drug) were obtained from Toronto Research Chemicals, Inc. (Toronto, ON, Canada). An NADPH regenerating system was purchased from BD Gentest Corp. (Woburn, MA, USA). Acetonitrile, dichloromethane, and formic acid were high-performance liquid chromatography grade. All other chemicals used were of analytical reagent grade or better.

\subsection{Plant Material}

P. ternate (Thunb.) Breit. (RPR, PRP, and PRPZA) were purchased from Huamiao Traditional Chinese Medicine Engineering Technology Development Center (Beijing, China) and confirmed by Institute of Chinese Materia Medica, China Academy of Chinese Medical Sciences.

\subsection{Animals}

Male Sprague-Dawley rats (180-220 g) were obtained by the Laboratory Animal Center of Guangzhou University of Chinese Medicine (Guangzhou, China; License: SCXK (yue) 2008-0020), and housed four per cage in a unidirectional airflow room under control (temperature: $23-25{ }^{\circ} \mathrm{C}$; relative humidity: $40 \%-70 \% ; 12 \mathrm{~h}$ light/dark cycle) with free access to standard rat food and water. All animal treatments followed guidelines for the care and use of laboratory animals and were approved by the ethics committee of Southern Medicine University (Guangzhou, China).

\subsection{Preparation of Herbs}

RPR, PRP or PRPZA (90 g) was immersed in $1800 \mathrm{~mL}$ of water for $30 \mathrm{~min}$ and then extracted with boiling water for $30 \mathrm{~min}$. The residue was again extracted with $900 \mathrm{~mL}$ of boiling water for $30 \mathrm{~min}$. The supernatant was combined and condensed to $500 \mathrm{~mL}$. The prepared water extracts $(10 \mu \mathrm{L})$ were diluted 100 times with methanol. The mixture was subsequently vortexed and then centrifuged at $18,000 \mathrm{~g}$ for $30 \mathrm{~min}$, and the supernatant was subjected to Ultra High Pressure Liquid Chromatography-mass spectrometry-Quadrupole-time of flight (UHPLC-MS-Q-TOF, Agilent Technologies, Santa Clara, CA, USA) for analysis. The detailed information of analysis conditions, the chromatograms and possible ingredients in RPR, PRP and PRPZA water extracts were shown in the supplementary materials.

\subsection{Preparation of Rat Liver Microsomes (RLMs)}

Rats were randomly assigned to eleven groups with ten animals in each group. After oral pretreatment with RPR, PRP or PRPZA water extracts $\left(1.8,0.9\right.$ and $0.45 \mathrm{~g} \cdot \mathrm{kg}^{-1} \cdot \mathrm{day}^{-1}$ as the high dose, medium dose and low dose, respectively), saline, or SKF-525A $\mathrm{HCl}\left(0.1 \mathrm{~g} \cdot \mathrm{kg}^{-1}\right)$ for 7 day, the rats were anaesthetized with ethyl carbamate $\left(50 \% \mathrm{w} / \mathrm{v}, 3 \mathrm{~mL} \cdot \mathrm{kg}^{-1}\right.$, i.p. $)$ on the eighth day, and the liver was excised. The livers of ten rats per group were combined to prepare RLMs. The detailed RLMs preparation procedures could be found in previous literature [38]. Bio-Rad protein assay (Bio-Rad, Hercules, CA, USA) was used to determine the concentration of microsomal protein with bovine serum albumin as the protein standard. 


\subsection{Determination of CYP3A Activity in RLMs by Tes Metabolism ex Vivo}

The detailed information about determination of CYP3A activity by Tes metabolism was described in our previous study [37]. In brief, an NADPH regenerating system, including Tes (4, 10, and $\left.30 \mu \mathrm{mol} \cdot \mathrm{L}^{-1}\right)$ and RLMs protein $\left(0.02-0.05 \mathrm{mg} \cdot \mathrm{mL}^{-1}\right)$, was created to evaluate the formation rate of metabolite $6 \beta-O H-T e s$ from Tes in RLMs with $7 \mathrm{~d}$ RPR, PRP or PRPZA pretreatment. The samples of Tes and $6 \beta-\mathrm{OH}-\mathrm{Tes}$ were analyzed by a validated UPLC method described in our published paper [39]. CYP3A activity ex vivo was expressed as nanomoles of $6 \beta-\mathrm{OH}-\mathrm{Tes}$ obtained per milligram of protein per minute. All experiments were run in triplicate.

\subsection{CYP3A Protein Expression of RLMs by Western Blot}

Eighty micrograms of total protein was separated by SDS-PAGE (4\% stacking gel, 10\% separating gel) and transferred from the gel to the PVDF membrane. Other detailed procedures were described in previous literature [37]. ECL chemiluminescence detection agent was applied to obtain the blot signals following the manufacturer's instructions. The relative intensity of the protein bands was scanned and quantified using Quantity One Program (Bio-Rad, Hercules, CA, USA).

\subsection{CYP3A1/2 mRNA Measurement by Real-Time PCR}

After pretreatment, rat hepatic total RNA was isolated using the TRIzol extraction method (Invitrogen, Carlsbad, CA, USA). cDNA was synthesized from total RNA using a reverse transcription kit (TaKaRa, Shiga, Japan). SYBR Green real-time PCR amplification and detection were then performed using an ABI 7500 system (Applied Biosystems, Foster City, CA, USA). The forward and reverse primers of CYP3A1, CYP3A2 and GAPDH were displayed in previous research [37], which also described the PCR mixture and thermal profile. Target relative mRNA levels were normalized against GAPDH mRNA levels. All samples were run in triplicate.

\subsection{Pharmacokinetics of BP in Rats with RPR, PRP or PRPZA Water Extracts Pretreatment for 7 days}

Rats were randomly assigned to five groups with five animals in each group. After pretreatment with RPR, PRP or PRPZA water extracts $\left(0.9 \mathrm{~g} \cdot \mathrm{kg}^{-1} \cdot \mathrm{day}^{-1}\right.$, p.o.) or saline for 7 days, the rats were given $\mathrm{BP} \cdot \mathrm{HCl}\left(0.5 \mathrm{mg} \cdot \mathrm{kg}^{-1}\right.$, single dose, i.v.). Serial blood samples were collected at $0,2,7,15,30$, $60,90,120,180,300,480$, and $720 \mathrm{~min}$ after $\mathrm{BP} \cdot \mathrm{HCl}$ administration. The plasma samples were processed and analyzed by a validated UPLC/MS/MS method as described in our previous studies [35,37]. CYP3A activity in vivo was determined by measuring the formation ratios of 6'-OH-BP and 1-PP from BP (6'-OH-BP/BP and 1-PP/BP ratio values).

\subsection{Data Analysis}

Pharmacokinetic parameters were calculated using the standard non-compartmental method by Practical Pharmacokinetic Program Version 97 (3P97). All results were presented as mean \pm standard deviation. Significant differences were analyzed using Student's $t$-test (two groups) or one-way 
ANOVA followed by LSD test (for more than two groups) by SPSS 19.0. Statistical differences were considered significant at $p<0.05$.

\section{Conclusions}

This study is the first to investigate the influence of RPR, as well as its processed products PRP and PRPZA, on CYP3A ex vivo and in vivo, which assessed the safety of co-administration of PR with other CYP3A-metabolizing drugs from the perspective of CYPs. Raw product RPR, as well as its processed products PRP and PRPZA can significantly inhibit CYP3A activity ex vivo. Such inhibition is due to the down-regulation of CYP3A protein and mRNA expression levels. Besides, the inhibitory effects caused by RPR and PRP ex vivo were more evident than that in vivo. Results of this study revealed that drug interaction between PR and the CYP3A-metabolizing drugs might be probable, suggesting that careful monitoring is essential for the concomitant use of PR with other drugs so as to avoid the adverse interactions. DDIs caused by PR-related co-therapy may be another factor that results in its toxicity. Considering no study has reported the index component in PR, so water extracts of PR were analyzed in this study based on clinical practices in China. Further research should be continued on the effects of bioactive ingredients in PR on CYP3A.

\section{Supplementary Materials}

Supplementary materials can be accessed at: http://www.mdpi.com/1420-3049/20/01/0792/s1.

\section{Acknowledgments}

This project was supported partly by the National Basic Research Program of China (973 Program) (2011CB505305), the Key International Joint Research Project of National Natural Science Foundation of China (81120108025) and the grant of National Natural Science Foundation of China (81473410).

\section{Author Contributions}

Linlin Lu, Zhongqiu Liu, Na Lin and Liang Liu designed the research; Jinjun $\mathrm{Wu}$, Zaixing Cheng, Shugui He, Jian Shi, Shuqiang Liu and Guiyu Zhang performed the study; Jinjun Wu, Zaixing Cheng and Lijun Zhu analyzed the data; Jinjun $\mathrm{Wu}$, Zaixing Cheng wrote and revised the manuscript; all authors read and approved the final version.

\section{Conflicts of Interest}

The authors declare no conflict of interest.

\section{References}

1. Ok, I.S.; Kim, S.H.; Kim, B.K.; Lee, J.C.; Lee, Y.C. Pinellia ternata, Citrus reticulata, and their combinational prescription inhibit eosinophil infiltration and airway hyperresponsiveness by suppressing CCR3+ and Th2 cytokines production in the ovalbumin-induced asthma model. Mediat. Inflamm. 2009, 2009, doi:10.1155/2009/413270. 
2. Kurata, K.; Tai, T.; Yang, Y.; Kinoshita, K.; Koyama, K.; Takahashi, K.; Watanabe, K.; Nunoura, Y. Quantitative analysis of anti-emetic principle in the tubers of Pinellia ternata by enzyme immunoassay. Planta Med. 1998, 64, 645-648.

3. Zou, Z.D.; Liu, N.; Guo, P.; Guo, L.Y.; Sun, Y.; Shi, J.; Wang, L. Analysis on clinical treatment in hypertension by traditional Chinese medicine for 10 years in Beijing. Zhongguo Zhong Yao Za Zhi 2007, 32, 1569-1572.

4. Wu, X.Y.; Zhao, J.L.; Zhang, M.; Li, F.; Zhao, T.; Yang, L.Q. Sedative, hypnotic and anticonvulsant activities of the ethanol fraction from Rhizoma Pinelliae Praeparatum. J. Ethnopharmacol. 2011, $135,325-329$.

5. Huang, C.; Zhang, X.S.; Zhu, R.R. Brief discussion on modern pharmaceutical research progress and compatibility changes of Radix Aconiti Lateralis Preparata and Rhizoma Pinelliae. China Pharm. 2012, 21, 19-20.

6. Zhou, S.S.; Ma, Z.C.; Liang, Q.D.; Wang, Y.G.; Tan, H.L.; Xiao, C.R.; Zhang, B.L.; Gao, Y. UPLC/Q-TOF-MS based chemical profiling approach to evaluate chemical composition of augmentation toxicity in combination of Radix Aconitiand Pinellia Praeparata. Acta Chim. Sin. 2012, 70, 284-290.

7. Wu, H.; Li, W.; Han, H.; Ji, R.; Ye, D.J. Studies on stimulating components of raw Pinellia ternata (Thunb.) (banxia). Zhongguo Zhong Yao Za Zhi 1999, 24, 763.

8. Zhong, L.Y.; Wu, H.; Zhang, K.W.; Wang, Q.R. Study on irritation of calcium oxalate crystal in raw Pinellia ternata. Zhongguo Zhong Yao Za Zhi 2006, 31, 1706-1710.

9. Zhong, L.Y.; Wu, H. Current researching situation of mucosal irritant compontents in Araceae family plants. Zhongguo Zhong Yao Za Zhi 2006, 31, 1561-1563.

10. Ge, X.Y.; Wu, H. phytochemical properties and quality evaluation methods of Pinelliae ternate. China Pharm. 2009, 18, 3-5.

11. Gonda, R.; Tomoda, M.; Shimizu, N.; Ohara, N.; Takagi, H.; Hoshino, S. Characterization of an acidic polysaccharide with immunological activities from the tuber of Pinellia ternata. Biol. Pharm. Bull. 1994, 17, 1549-1553.

12. Tomoda, M.; Gonda, R.; Ohara, N.; Shimizu, N.; Shishido, C.; Fujiki, Y. A glucan having reticuloendothelial system-potentiating and anti-complementary activities from the tuber of Pinellia ternata. Biol. Pharm. Bull. 1994, 17, 859-861.

13. Yang, B.Y.; Li, M.; Shi, J.; Xia, D.M.; Li, X.X.; Yang, X.Y. A system research on HPLC characteristic fingerprint of Pinelliae Rhizoma and Pinelliae Rhizoma Praeparatum cum Zingibere et Alumine. Chin. Tradit. Herb. Drugs 2014, 45, 652-658.

14. Cheng, Z.X.; Wu, J.J.; Liu, Z.Q.; Lin, N. Development of a hydrophilic interaction chromatography-UPLC assay to determine trigonelline in rat plasma and its application in a pharmacokinetic study. Chin. J. Nat. Med. 2013, 11, 164-170.

15. Xiu, Y.F.; Hong, X.K.; Wang, Z.H. Progress of studies on preparation of Rhizoma Pinelliae. Chin. Tradit. Patent Med. 2004, 26, 38-40.

16. Cai, B.C.; Qin, K.M.; Wu, H.; Cai, H.; Lu, T.L.; Zhang, X.D. Chemical mechanism during Chinese medicine processing. Prog. Chem. 2012, 24, 637-649.

17. Pei, M.; Duan, X.; Pei, X. Compatability chemistry of acid-alkaline pair medicine of Fuzi and Gancao in Sini decoction. Zhongguo Zhong Yao Za Zhi 2009, 34, 2047-2050. 
18. Zhang, J.M.; Liao, W.; He, Y.X.; He, Y.; Yan, D.; Fu, C.M. Study on intestinal absorption and pharmacokinetic characterization of diester diterpenoid alkaloids in precipitation derived from fuzi-gancao herb-pair decoction for its potential interaction mechanism investigation. J. Ethnopharmacol. 2013, 147, 128-135.

19. Soderberg Lofdal, K.C.; Andersson, M.L.; Gustafsson, L.L. Cytochrome P450-mediated changes in oxycodone pharmacokinetics/pharmacodynamics and their clinical implications. Drugs 2013, 73, 533-543.

20. Yee, D.A.; Atayee, R.S.; Best, B.M.; Ma, J.D. Observations on the urine metabolic profile of codeine in pain patients. J. Anal. Toxicol. 2014, 38, 86-91.

21. Tian, X.; Cheng, Z.Y.; He, J.; Jia, L.J.; Qiao, H.L. Concentration-dependent inhibitory effects of baicalin on the metabolism of dextromethorphan, a dual probe of CYP2D and CYP3A, in rats. Chem. Biol. Interact. 2013, 203, 522-529.

22. Chang, S.Y.; Fancher, R.M.; Zhang, H.; Gan, J., Mechanism-based inhibition of human cytochrome P4503A4 by domperidone. Xenobiotica 2010, 40, 138-145.

23. Wojcikowski, J.; Boksa, J.; Daniel, W.A. Main contribution of the cytochrome P450 isoenzyme 1A2 (CYP1A2) to N-demethylation and 5-sulfoxidation of the phenothiazine neuroleptic chlorpromazine in human liver-A comparison with other phenothiazines. Biochem. Pharmacol. 2010, 80, 1252-1259.

24. Ishiguro, N.; Senda, C.; Kishimoto, W.; Sakai, K.; Funae, Y.; Igarashi, T. Identification of CYP3A4 as the predominant isoform responsible for the metabolism of ambroxol in human liver microsomes. Xenobiotica 2000, 30, 71-80.

25. Izzo, A.A.; Ernst, E. Interactions between herbal medicines and prescribed drugs: An updated systematic review. Drugs 2009, 69, 1777-1798.

26. Saxena, A.; Tripathi, K.P.; Roy, S.; Khan, F.; Sharma, A. Pharmacovigilance: Effects of herbal components on human drugs interactions involving cytochrome P450. Bioinformation 2008, 3, 198-204.

27. Yoo, H.H.; Kim, S.A.; Kim, I.S.; Ko, S.G. The Evaluation of CP-001 (a standardized herbal mixture of Houttuynia cordata, Rehmannia glutinosa, Betula platyphylla, and Rubus coreanus) for cytochrome P450-related herb-drug interactions. Evid.-Based Complement. Altern. Med. 2013, 2013, doi:10.1155/2013/824270.

28. Chen, X.W.; Sneed, K.B.; Pan, S.Y.; Cao, C.; Kanwar, J.R.; Chew, H.; Zhou, S.F. Herb-drug interactions and mechanistic and clinical considerations. Curr. Drug Metab. 2012, 13, 640-651.

29. Sugiyama, M.; Fujita, K.; Murayama, N.; Akiyama, Y.; Yamazaki, H.; Sasaki, Y. Sorafenib and sunitinib, two anticancer drugs, inhibit CYP3A4-mediated and activate CY3A5-mediated midazolam 1'-hydroxylation. Drug Metab. Dispos. 2011, 39, 757-762.

30. Ingelman-Sundberg, M. Pharmacogenetics of cytochrome P450 and its applications in drug therapy: The past, present and future. Trends Pharmacol. Sci. 2004, 25, 193-200.

31. Martignoni, M.; Groothuis, G.M.; de Kanter, R. Species differences between mouse, rat, dog, monkey and human CYP-mediated drug metabolism, inhibition and induction. Expert Opin. Drug Metab. Toxicol. 2006, 2, 875-894. 
32. Shou, M.; Lu, T.; Krausz, K.W.; Sai, Y.; Yang, T.; Korzekwa, K.R.; Gonzalez, F.J.; Gelboin, H.V. Use of inhibitory monoclonal antibodies to assess the contribution of cytochromes $\mathrm{P} 450$ to human drug metabolism. Eur. J. Pharmacol. 2000, 394, 199-209.

33. Friedrich, G.; Rose, T.; Rissler, K. Determination of testosterone metabolites in human hepatocytes. I. Development of an on-line sample preparation liquid chromatography technique and mass spectroscopic detection of 6beta-hydroxytestosterone. J. Chromatogr. B Anal. Technol. Biomed. Life Sci. 2003, 784, 49-61.

34. Chen, X.M.; Wei, M.; Zhang, H.M.; Luo, C.H.; Chen, Y.K.; Chen, Y. Effect of vanillin and ethyl vanillin on cytochrome P450 activity in vitro and in vivo. Food Chem. Toxicol. 2012, 50, 1897-1901.

35. Zhu, L.; Yang, X.; Zhou, J.; Tang, L.; Xia, B.; Hu, M.; Zhou, F.; Liu, Z. The exposure of highly toxic aconitine does not significantly impact the activity and expression of cytochrome P450 $3 \mathrm{~A}$ in rats determined by a novel ultra performance liquid chromatography-tandem mass spectrometric method of a specific probe buspirone. Food Chem. Toxicol. 2013, 51, 396-403.

36. Debri, K.; Boobis, A.R.; Davies, D.S.; Edwards, R.J. Distribution and induction of CYP3A1 and CYP3A2 in rat liver and extrahepatic tissues. Biochem. Pharmacol. 1995, 50, 2047-2056.

37. Wu, J.; Cheng, Z.; Zhu, L.; Lu, L.; Zhang, G.; Wang, Y.; Xu, Y.; Lin, N.; Liu, Z. Coadministration of Pinellia ternata can significantly reduce Aconitum carmichaelii to inhibit CYP3A activity in Rats. Evid.-Based Complement. Altern. Med. 2014, 2014, doi:10.1155/2014/734867.

38. Chen, J.; Halls, S.C.; Alfaro, J.F.; Zhou, Z.; Hu, M. Potential beneficial metabolic interactions between tamoxifen and isoflavones via cytochrome P450-mediated pathways in female rat liver microsomes. Pharm. Res. 2004, 21, 2095-2104.

39. Wu, J.J.; Cheng, Z.X.; Liu, Z.Q.; Lin, N., Influence of Wutou Tang on Activity of CYP3A4 in Rat Liver Microsomes. Chin. J. Exp. Tradit. Med. Formul. 2013, 19, 233-236.

Sample Availability: Samples of the RPR, PRP and PRPZA herbs are available from the authors.

(C) 2015 by the authors; licensee MDPI, Basel, Switzerland. This article is an open access article distributed under the terms and conditions of the Creative Commons Attribution license (http://creativecommons.org/licenses/by/4.0/). 